\title{
Transcriptome sequencing and analysis during seed growth and development in Euryale ferox Salisb
}

\author{
Xian Liu', Zhen He ${ }^{1} \mathbb{B}$, Yulai Yin², Xu Xu', Weiwen $\mathrm{Wu}^{1}$ and Liangjun $\mathrm{Li}^{i^{*}}$
}

\begin{abstract}
Background: Euryale ferox Salisb., an annual aquatic plant, is the only species in the genus Euryale in the Nymphaeaceae. Seeds of E. ferox are a nutritious food and also used in traditional Chinese medicine (Qian Shi in Mandarin). The molecular events that occurred during seed development in E. ferox have not yet been characterized. In this study, we performed transcriptomic analysis of four developmental stages $(T 1, T 2, T 3$, and $T 4)$ in $E$. ferox seeds with three biological replicates per developmental stage to understand the physiological and biochemical processes during $E$. ferox seeds development.

Results: $313,844,425$ clean reads were assembled into 160,107 transcripts and 85,006 unigenes with N50 lengths of 2052 bp and 1399 bp, respectively. The unigenes were annotated using five public databases (NR, COG, Swiss-Prot, KEGG, and GO). In the KEGG database, all of the unigenes were assigned to 127 pathways, of which phenylpropanoid biosynthesis was associated with the synthesis of secondary metabolites during E. ferox seed growth and development. Phenylalanine ammonia-lyase (PAL) as the first key enzyme catalyzed the conversion of phenylalanine to trans-cinnamic acid, then was related to the synthesis of flavonoids, lignins and alkaloid. The expression of PAL1 reached its peak at T3 stage, followed by a slight decrease at T4 stage. Cytochrome P450 (P450), encoded by CYP84A1 (which also called ferulate-5-hydroxylase (F5H) in Arabidopsis), was mainly involved in the biosynthesis of lignins.

Conclusions: Our study provides a transcriptomic analysis to better understand the morphological changes and the accumulation of medicinal components during E. ferox seed development. The increasing expression of PAL and P450 encoded genes in phenylpropanoid biosynthesis may promote the maturation of E. ferox seed including size, color, hardness and accumulation of medicinal components.
\end{abstract}

Keywords: Euryale ferox Salisb., Seed development, Transcriptome, Phenylpropanoid biosynthesis, Phenylalanine ammonia-lyase, Cytochrome P450

\section{Background}

Euryale ferox Salisb., an annual aquatic herbaceous plant, is the only species in the genus Euryale in the botanical family Nymphaeaceae [1, 2]. E. ferox is known commonly as fox nut or gorgon nut [3], and widely distributed in tropical and subtropical regions of east and southeast Asia [4]. E. ferox is usually grown in lakes, ponds, reservoirs, and other shallow bodies of water $[4$, 5]. Unlike most other aquatic crops, the floating leaves

\footnotetext{
* Correspondence: ljil@yzu.edu.cn

${ }^{1}$ School of Horticulture and Plant Protection, Yangzhou University, 48 Wenhui East Road, Yangzhou, Jiangsu Province 225009, People's Republic of China

Full list of author information is available at the end of the article
}

of $E$. ferox are comparatively large, reaching up to $1.5 \mathrm{~m}$ in diameter [5] (Fig. 1). The seeds of E. ferox are rich in starch, proteins, vitamins, minerals and many other nutritional ingredients [6, 7]. The "Compendium of Materia Medica", a book of Chinese traditional medicine written during the Ming dynasty (1368-1644), describes the many medicinal uses of $E$. ferox, which included its use as a kidney tonic, for nourishing the spleen, as a treatment for diarrhea, as well as for eliminating dampness [8]. Seeds of E. ferox are also a significant component of contemporary Chinese herbal medicine and are used to treat a variety of diseases, such as kidney failure, chronic diarrhea, excessive leucorrhea, and hypofunction of the spleen $[9,10]$. At present, studies of E. ferox are 


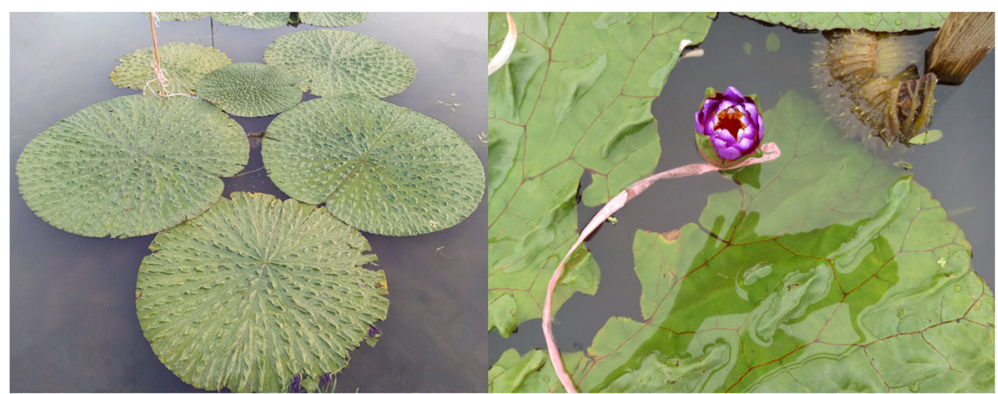

Fig. 1 The leaves and flower of Euryale ferox Salisb. in shallow body of water

focused on seed dormancy and germination traits [4], genetic diversity [11], and the antioxidant activity in the seeds $[3,10,12,13]$. However, there are few reports describing the stages of seed development in E. ferox in the literature. It is particularly important for seeds to explore the morphological changes and the accumulation of medicinal components.

Phenylpropanoid biosynthesis involved in numerous important biological processes, such as detoxification of xenobiotics and synthesis of secondary metabolites [14, 15], plays an important role in development of many plant. Phenylalanine ammonia-lyase (PAL) [14] and cytochrome P450 (P450) [16] both encoded by a multi-gene family with different functions [17-22] are key enzymes in the proceed of phenylpropanoid biosynthesis. The expression of PALs possesses tissue-specific in Arabidopsis thaliana [23] and Salvia miltiorrhiza [14], among which the highest expression of PAL1 and PAL2 in root and mature flower of Arabidopsis thaliana [23], PAL1, PAL2 and PAL3 with highest expression in root, stem and leaf of Salvia miltiorrhiza, respectively [14]. The expression of FaPAL6 increased during the strawberry fruit riping along with the anthocyanin accumulation affecting fruit color [24]. For P450 genes (cytochrome P450, CYP), the expression of $C a C Y P 94 B 1$ was up-regulated, whereas the expressions of $C a C Y P 72 A 15, C a C Y P 701 A 3$ and $C a C Y P 71 A 25$ were down-regulated in the perisperm at three developmental stages of Coffea Arabica [25]. In addition, TaCYP78A3 was specifically expressed in reproductive organs and influenced the size of wheat seed [26]. E. ferox seed, as an important medicinal component, was closely related to many secondary metabolites, such as flavonoids, lignins and alkaloid [14]. Phenylpropanoid biosynthesis involved in the synthesis of secondary metabolites was selected to analyse the relationship with E. ferox seed development, and PAL and P450 are the vital differential genes in this pathway that we found.

In this study, we performed a transcriptomic analysis of $E$. ferox seed at four developmental stages $(10,20,30$, and 40 days after anthesis) on the basis of morphological characteristics $[27,28]$. Quantitative real-time PCR on the basis of six genes associated with nutritional and functional substances of E. ferox seed validated the transcriptome data. Two unigenes -PAL and P450 encoded genes associated with phenylpropanoid biosynthesis during E. ferox seed development were analysed to investigating the relationship with morphological changes and the accumulation of medicinal components.

\section{Methods \\ Plant material and sample collection}

Euryale ferox plants were collected from Suzhou of Jiangsu Province, China. Specifically, seeds of E. ferox begin to swell at 10 days after anthesis, and grow rapidly from 20 to 30 days after anthesis, while reach physiological maturity at 40 days after anthesis (Fig. 2). So the seed samples used for cDNA library construction were collected on 10, 20, 30, and 40 days after anthesis (stages $\mathrm{T} 1, \mathrm{~T} 2, \mathrm{~T} 3$, and $\mathrm{T} 4$, respectively), with three biological replicates per developmental stage. All samples were frozen in liquid nitrogen immediately upon collection and stored at $-80^{\circ} \mathrm{C}$.

\section{RNA preparation and CDNA library construction}

Total RNA was extracted from the 12 samples separately. RNA contamination and degradation was detected by electrophoresis on $1 \%$ agarose gels. RNA purity was measured with a NanoPhotometer ${ }^{\circ}$ spectrophotometer (IMPLEN, CA, USA), and RNA concentration was determined with a Qubit ${ }^{\circ}$.0 Flurometer and the Qubit $^{\circ}$ RNA Assay Kit (Life Technologies, CA, USA). RNA integrity was evaluated using the Nano 6000 Assay Kit on the Agilent Bioanalyzer 2100 system (Agilent Technologies, CA, USA).

To construct the cDNA libraries, we used $3 \mu \mathrm{g}$ total RNA per sample as the input material. The NEBNext ${ }^{\circ}$ Ultra $^{\mathrm{Tm}}$ RNA Library Prep Kit for Illumina ${ }^{\circ}$ (NEB, USA) was used to generate a series of corresponding libraries, and index codes were applied to every sample. Briefly, mRNA was purified from total RNA using oligo dT magnetic beads. RNA fragmentation was carried out at high temperature in the presence of divalent cations in 


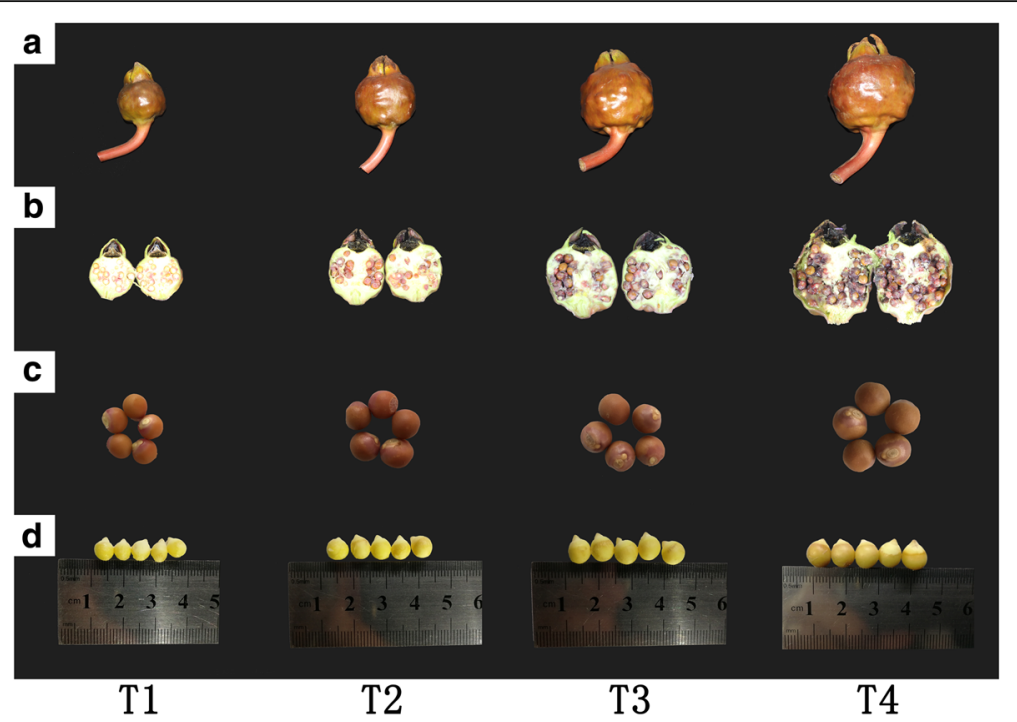

Fig. 2 The fruit, the longitudinal section of fruit, seed and kernel of Euryale ferox Salisb. during four development stages (T1, T2, T3 and T4). a Fruit; (b) The longitudinal section of fruit; (c) Seed; (d) Kernel

NEBNext First Strand Synthesis Reaction Buffer (5X). First-strand cDNA was synthesized using random hexamer primers and M-MLV Reverse Transcriptase (RNase $\mathrm{H}^{-}$). Second strand was synthesized using DNA Polymerase I and RNase $\mathrm{H}$. The exonuclease/polymerase activities were used to convert the remaining singlestranded overhangs to blunt ends. After adenylation of the $3^{\prime}$ ends of the DNA fragments, the NEBNext Adaptor containing a hairpin loop structure was added. cDNA fragments between 150 and $200 \mathrm{bp}$ were purified using the AMPure XP system (Beckman Coulter, Beverly, USA), and the cDNA library was non-strand specific. Afterwards, the $3 \mu$ U USER Enzyme (NEB, USA) was made use of picking size, adaptor-ligated cDNA at $37^{\circ} \mathrm{C}$ for $15 \mathrm{~min}$ followed by $5 \mathrm{~min}$ at $95^{\circ} \mathrm{C}$ before PCR. PCR was performed using universal PCR primers, Index (X) Primer and Phusion High-Fidelity DNA polymerase. The Agilent Bioanalyzer 2100 system was used to purify the PCR products and to evaluate the quality of the cDNA libraries. Paired-end DNA sequencing data for the 12 libraries was generated on an Illumina Hiseq 2000 instrument.

\section{De novo CDNA assembly and functional annotation}

To obtain clean reads, sequencing adaptors and lowquality reads were removed from each library. For increasing the amount of date to reduce the unigene redundancy, and ensuring the low expression genes assembled more completely, the remaining clean reads were adopted the method of the merged assembly of Trinity software [29]. Specifically, the clean reads in each library were first assembled into contigs, then the contigs were clustered to form unigenes. The clean reads were mapped back to the homologous contigs based on the paired-end reads, and the different contigs from their transcripts and the distance were also distinguished by the methods of the De Bruijn and sequencing information.

The unigene sequences were annotated using the following public databases: NCBI non-redundant protein sequences (NR) [30], Clusters of Orthologous Groups (COG) [31], Swiss-Prot [32], Kyoto Encyclopedia of Genes and Genomes (KEGG) [33], and Gene Ontology (GO) [34]. The unigene sequences were aligned using BLASTX with an E-value of $<10^{-5}$. If results from the different databases were mutually contradictory, a priority order of NR, Swiss-Prot, KEGG, and COG was followed to decide the annotation. Nevertheless, if a unigene did not align to any of the databases, ESTScan software was chosen to infer the direction of transcription [35].

\section{Differential gene expression analysis}

Each of the 12 E. ferox seed cDNA libraries was aligned separately to the transcriptome assemblies using Bowtie [36]. The counting of alignments was estimated using the RSEM package [37]. The RPKM method was then used to calculate the numbers of differentially expressed genes (DEGs) from pairwise comparisons of the four seed developmental stages [38]. From the biological replicates in this study, DESeq [39] implemented in $\mathrm{R}$ package was used to analyze the differential expression between two groups. $P$ values were adjusted using the Benjamini-Hochberg approach to control the false discovery rate (FDR). Genes with an adjusted $P$-value $<0.05$ found by DESeq were usually identified as DEGs. However, genes with FDR $<0.01$ and FC (Fold Change) $\geq 2$ 
Table 1 Summary of sample information and transcriptome sequencing output statistics for the E. ferox seed RNA-seq libraries

\begin{tabular}{|c|c|c|c|c|c|c|}
\hline Sample & Date of Collection & Total Clean Reads & Total Clean Nucleotides (nt) & GC Percent (\%) & N Percent (\%) & Q30 Percent (\%) \\
\hline$\overline{1-1}$ & 20, August, 2016 & $22,421,428$ & $6,651,313,472$ & 49.27 & 0 & 91.98 \\
\hline $1-2$ & 20, August, 2016 & $28,358,306$ & $8,449,902,688$ & 49.06 & 0 & 91.69 \\
\hline $1-3$ & 20, August, 2016 & $21,297,839$ & $6,334,599,486$ & 49.00 & 0 & 91.71 \\
\hline $2-1$ & 30, August, 2016 & $24,816,321$ & $7,393,759,062$ & 48.80 & 0 & 91.18 \\
\hline $2-2$ & 30, August, 2016 & $25,714,239$ & $7,656,559,222$ & 49.68 & 0 & 92.62 \\
\hline $2-3$ & 30, August, 2016 & $23,400,093$ & $6,976,232,182$ & 49.94 & 0 & 91.10 \\
\hline $3-1$ & 09,September, 2016 & $22,236,747$ & $6,619,274,594$ & 50.96 & 0 & 91.87 \\
\hline $3-2$ & 09,September, 2016 & $24,450,792$ & $7,274,216,700$ & 50.79 & 0 & 92.11 \\
\hline $3-3$ & 09,September, 2016 & $27,901,420$ & $8,307,711,474$ & 49.20 & 0 & 92.09 \\
\hline $4-1$ & 19,September, 2016 & $28,587,601$ & $8,504,756,010$ & 50.28 & 0 & 92.56 \\
\hline $4-2$ & 19,September, 2016 & $29,452,392$ & $8,769,841,382$ & 50.83 & 0 & 92.16 \\
\hline $4-3$ & 19,September, 2016 & $35,207,247$ & $10,466,146,780$ & 50.73 & 0 & 92.42 \\
\hline
\end{tabular}

served as standards in the screening, and FC represents the specific value between two samples. In addition, the phylogenetic relationships of $\mathrm{P} 450$ and beta-glucosidase genes were assessed using the neighbor-joining (NJ) method in MEGA version 7.0 [40].

\section{Quantitative real-time PCR analysis}

Total RNA was extracted in triplicate from the four developmental stages of $E$. ferox seeds (total of 12 samples) using Trizol $^{\circ}$ Reagent (Invitrogen, USA), and then reversed transcribed into cDNA using a PrimeScript ${ }^{\circ} \mathrm{RT}$ reagent Kit (Takara, Dalian, China). Six differentially expressed genes associated with nutritional and functional substances in E. ferox seed were selected to validate the transcriptome data using quantitative real-time PCR. The gene-specific primers for the six unigene sequences were designed with Primer Premier 5 software [41], and the $\beta$-Actin gene was used as an internal gene expression control: the gene was amplified with forward primer 5'-GACTCTGGTGATGGTGT-3' and reverse primer 5'-CACTTCATGATGGAGTTGT-3'. The amplifications were performed in $20 \mu \mathrm{l}$ reactions consisting of $10 \mu \mathrm{l} 2 \times \mathrm{SYBR}^{\circ}$ Premix EX Taqll (Tli RNaseH
Plus) (TaKaRa, Dalian, China), $2.0 \mu \mathrm{l}$ of a mixture of the forward and reverse primers, $2.0 \mu \mathrm{l}$ cDNA template, and $6.0 \mu \mathrm{l}$ sterile $\mathrm{dH}_{2} \mathrm{O}$. The amplifications were performed on a CFX-96 real-time PCR system (Bio-Rad) using the following quantitative real-time PCR program: $95{ }^{\circ} \mathrm{C}$ for $3 \mathrm{~min}$, followed by 39 cycles of $95{ }^{\circ} \mathrm{C}$ for $30 \mathrm{~s}$ and $\mathrm{Tm}$ for $30 \mathrm{~s}$. Each amplification reaction was performed in triplicate.

\section{Results}

\section{Collection of Euryale ferox seed}

Transcriptome sequencing was used to analyze the complex physiological and biochemical processes that occurred during four developmental stages of Euryale ferox seeds. The first stage was designated as 10 days after anthesis with obvious small size fruit and seeds. The other three stages were 20,30, and 40 days after anthesis. The fruit and seeds gradually became larger along with the color deepening and mechanical hardening of seeds (Fig. 2). Because three biological replicates were carried out for each developmental stage, a total of 12 samples were used in the analysis of seed development. Specific information of 12 samples is shown in Table 1.

Table 2 Summary statistics for the assembled transcripts and unigenes

\begin{tabular}{lllll}
\hline Length range & Transcripts & Transcripts percent (\%) & Unigenes & Unigenes percent (\%) \\
\hline $200-300$ & 34,006 & $21.24 \%$ & 30,871 & $36.32 \%$ \\
$300-500$ & 26,830 & $16.76 \%$ & 20,051 & $23.59 \%$ \\
$500-1000$ & 32,751 & $20.46 \%$ & 16,287 & $19.16 \%$ \\
$1000-2000$ & 35,975 & $22.47 \%$ & 10,154 & $11.95 \%$ \\
$2000+$ & 19,544 & & 7642 & $8.99 \%$ \\
Total number & 160,107 & & 85,006 & $66,000,704$ \\
Total length & $193,603,846$ & 1399 & \\
N50 length & 2052 & & 776.42 & \\
Mean length & 1209.22 & & \\
\hline
\end{tabular}




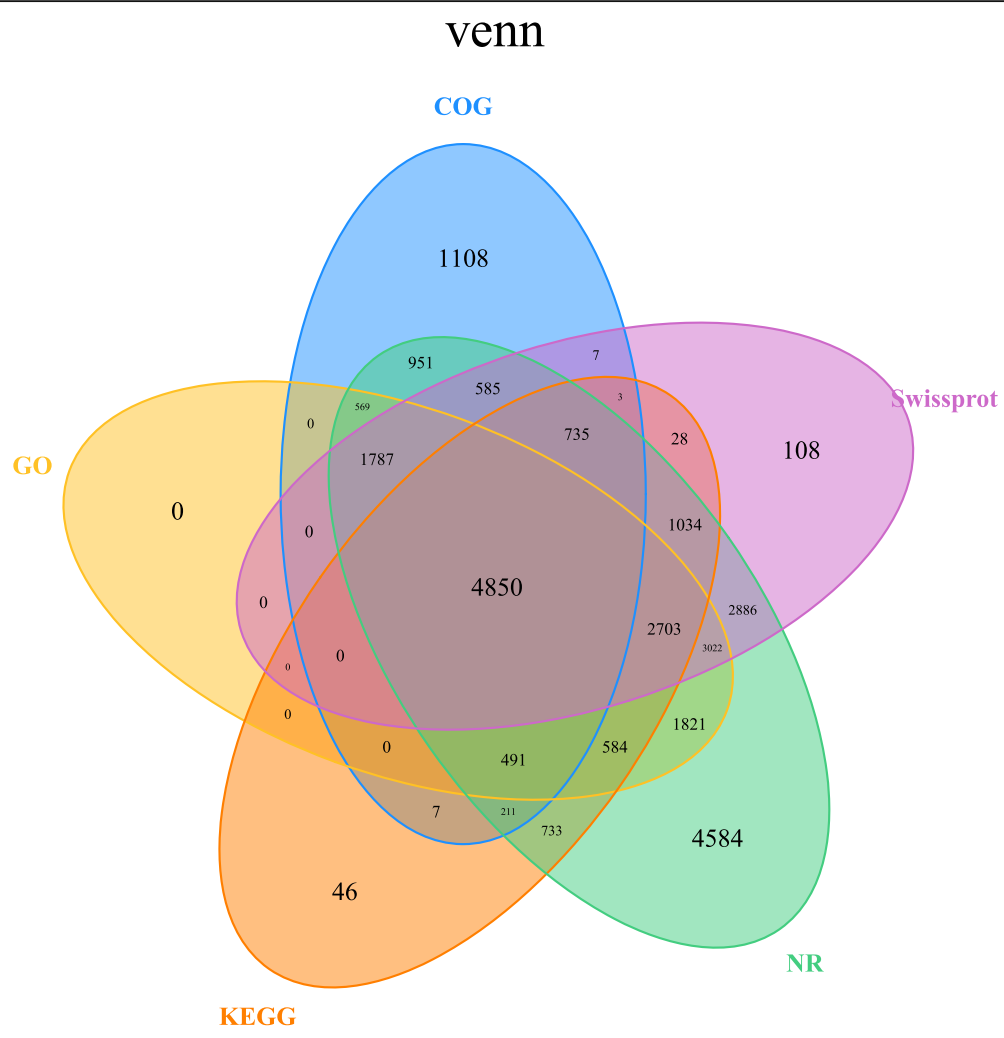

Fig. 3 Venn diagram of Euryale ferox Salisb. seed unigenes. Functional annotation was done using five public databases; NCBI non-redundant protein sequences (NR), Swiss-Prot, COG, Gene Ontology (GO), and Kyoto Encyclopedia of Genes and Genomes (KEGG). The shared unigenes are indicated in the intersections

\section{RNA-seq and de novo transcriptome assembly}

In order to obtain comprehensive and effective transcriptomic information for E. ferox seed growth and development, $12 \mathrm{cDNA}$ libraries were constructed and generated paired-end sequence reads using the Illumina Hiseq 2000 platform. Detailed information about the transcriptome sequencing is given in Table 1 . A total of $313,844,425$ clean reads originated from the 12 E. ferox cDNA libraries were obtained following removal of adaptors and low-quality reads from the libraries (the transcriptome data is being submitted to SRA database). The percent $\mathrm{G}+\mathrm{C}$, percentage of Ns (unknown bases), and fraction of bases with quality scores of Q30 for the 12 libraries averaged $48.8 \%, 0 \%$, and $91.1 \%$, respectively. These numbers showed that the data was of high quality. Trinity software was then used to assemble the clean reads into 160,107 transcripts with N50 length of 2052 bp and 85,006 unigenes with N50 length of 1399 bp. The relative lengths of the assembled sequences is one standard by which a transcriptome assembly can be assessed. For the present study, the summary statistics of the assembled transcripts and unigenes are shown in Table 2. Of these, assembled sequences $<200 \mathrm{bp}$ were not taken into account. In the final assemblies there were 60,836 transcripts ranging from 200 to 500 bp in length, which accounted for $38 \%$ of the total. There were $32,751(20.46 \%)$ and 66,519 (41.55\%) transcripts from 500 to $1000 \mathrm{bp}$ and > $1000 \mathrm{bp}$ in length, respectively. Compared with the assembled transcripts, the assembled unigenes in the above three length ranges accounted for $59.91 \%, 19.16 \%$, and $20.94 \%$ of the total (Table 2). In addition, the assembled sequences were also annotated by BLASTX against Arabidopsis thaliana TAIR10 (Additional file 3: Table S1) and rice 7.0 proteomes (Additional file 4: Table S2).

\section{Annotation and functional classification}

The assembled sequences were used as queries in BLAST searches $\left(\mathrm{E} \leq 10^{-5}\right)$ against the NR, COG, SwissProt, KEGG, and GO databases, and the results are shown in Fig. 3. The majority of the unigenes $(27,546$; $32.4 \%)$ were annotated from the NR database. In contrast, only 11,304 annotated unigenes were matched to the COG database. As shown in Fig. 3, a total of 4850 unigenes were annotated with all five databases.

To further utilize the transcriptome data, the COG, GO, and KEGG databases were used to identify the molecular processes that occur during the growth and development of $E$. ferox seed. The annotated unigenes were initially classified into 25 COG categories with the 


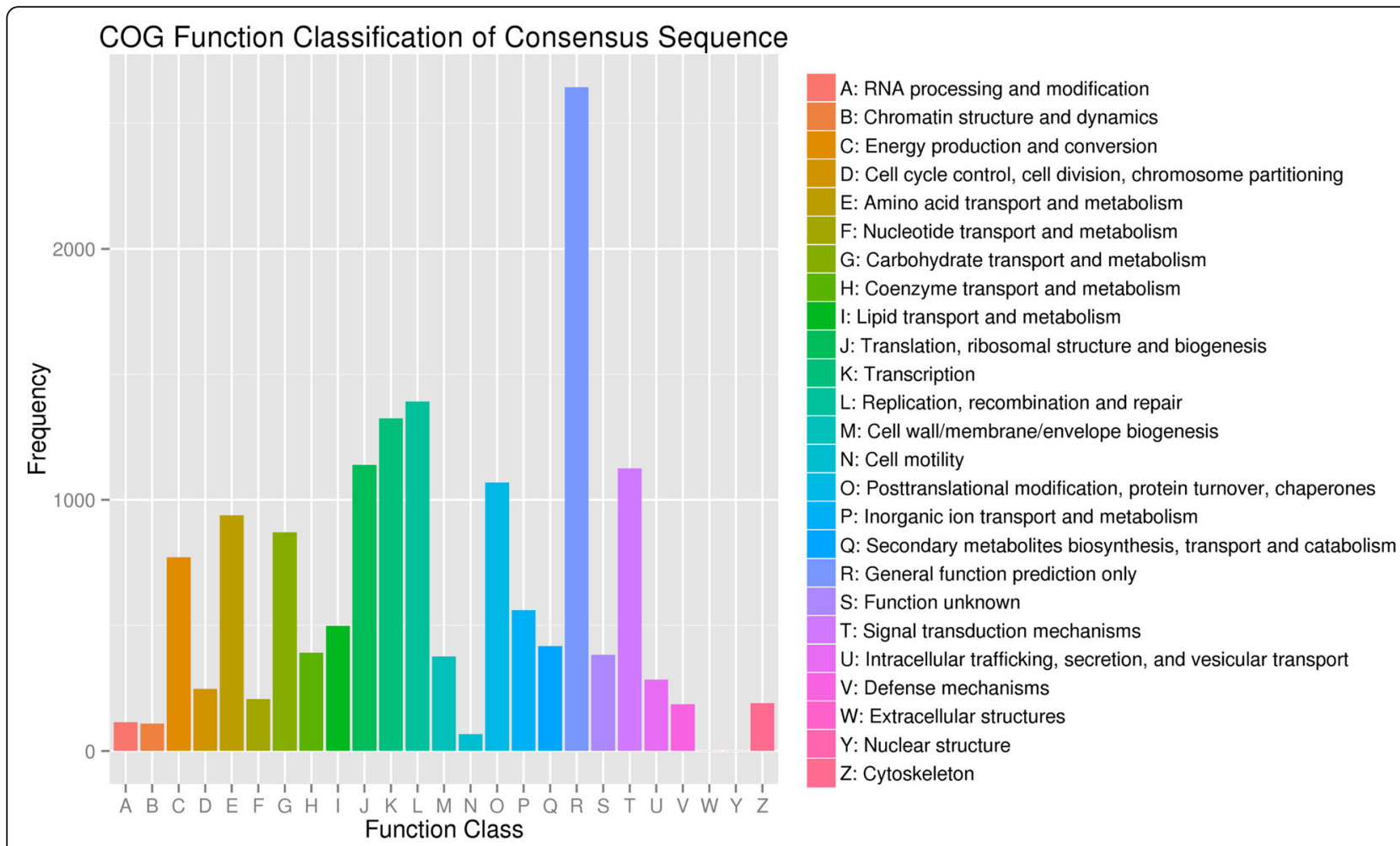

Fig. 4 Histogram of clusters of orthologous groups (COG) classification. A total of 11,304 unigenes with lengths more than 300 bp were divided into 25 COG categories

largest group being "General function prediction only" (2644; 23.39\%) (Fig. 4). Genes in "Carbohydrate transport and metabolism" $(870,7.70 \%)$ and "Secondary metabolites biosynthesis, transport and catabolism" (417, $3.69 \%$ ) are involved in biosynthesis of the nutritional and functional substances which benefit human health. The Gene Ontology (GO), which is an internationally standardized classification system, was used to assign 15,827 unigenes from the four seed developmental stages to the three principal GO domains; "cellular component", "molecular function", and "biological process" (Fig. 5). In the "cellular component" category, "cell" and "cell part" were the most abundant terms. For the category of "molecular function", "catalytic activity" and "binding" were the most prominent. "Metabolic process" and "cellular process" were the most abundant terms in the "biological process" category.

\section{Differential gene expression and pathway enrichment analysis}

Transcriptome sequencing is a powerful and effective approach that is often used to analyze gene expression at the whole genome level without a reference genome sequence, and this method can also be taken to identify a variety of metabolic processes [42-44]. In this study, a total of 4897 DEGs were confirmed among the four developmental stages of E. ferox seed. Specific information describing the up- and down-regulated DEGs identified in pairwise comparisons between the four seed developmental stages is shown in Additional file 5: Table S3. The KEGG database is used to systematically map unigenes to specific metabolic pathways and to describe the function of the unigenes. In the present study, all of the unigenes were assigned to 127 pathways which were divided into five groups: cellular processes, environmental information processing, genetic information processing, metabolism, and organismal systems (Additional file 1: Figure S1). Of the five groups, the metabolism group contained the largest number of pathways. The most abundant pathway was "ribosome" which had 539 unigenes, followed by "carbon metabolism" with 515 genes, and "biosynthesis of amino acids" with 470 genes.

\section{Phenylpropanoid pathway}

In the phenylpropanoid pathway of E. ferox seed development, PAL is the first key enzyme that catalyzed the conversion of phenylalanine to trans-cinnamic acid, then experienced a series of complex channels to finally synthesizes some important secondary metabolites including flavonoids, lignins and alkaloid (Fig. 6). PAL in E. ferox seed was encoded by c55946.graph_c2, which shared highest identities (79\%) with PAL1 gene of 


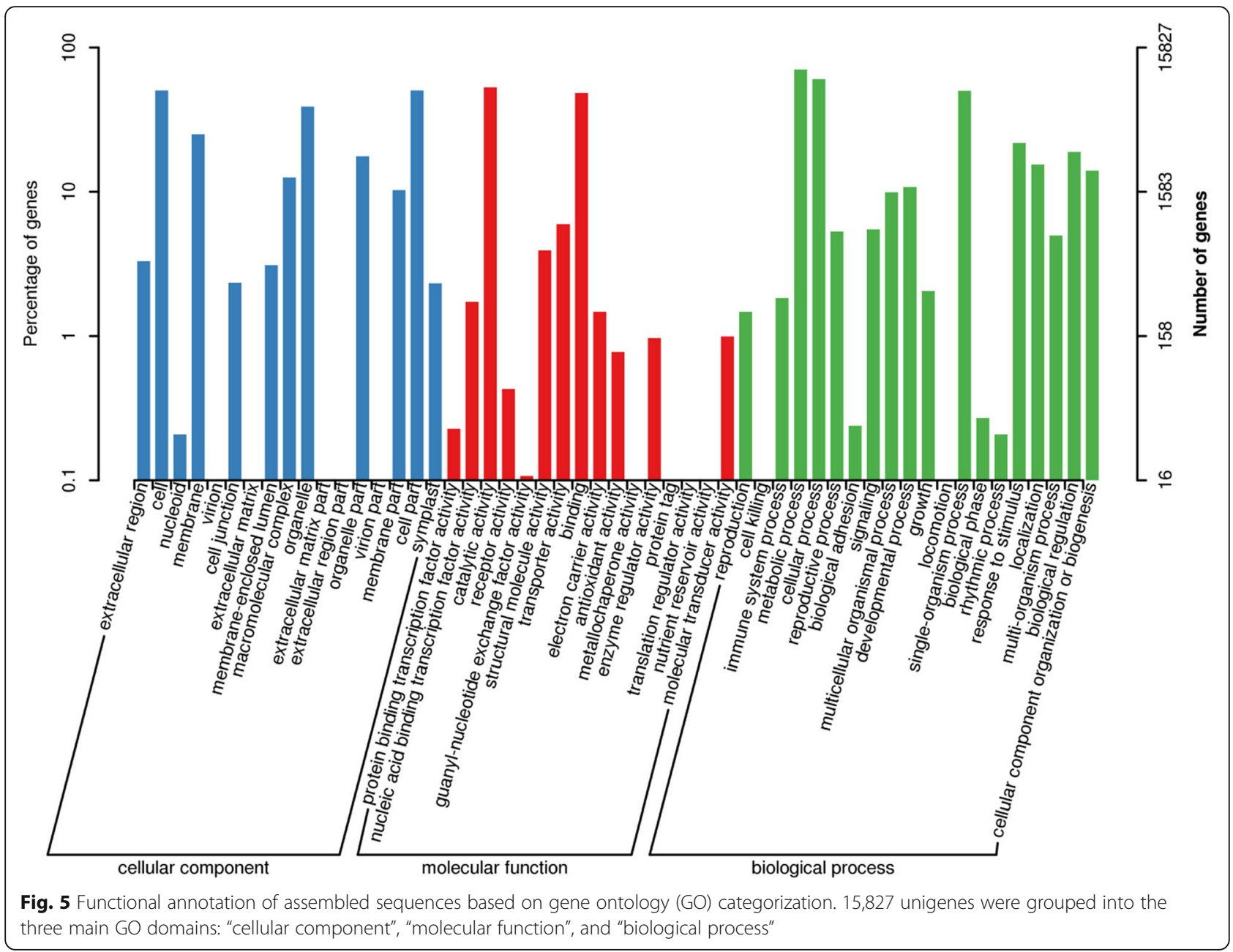

Myrtaceae in GenBank. During E. ferox seed growth and development, the expression of c55946.graph_c2 reached its peak at T3 stage, followed by a slight decrease at T4 stage. Another key enzyme is $\mathrm{P} 450$ encoded by c17722.graph_c0, which shared highest identities (70\%) with CYP84A1 gene of Ananas comosus in GenBank. And the CYP84A1 gene was also called ferulate-5hydroxylase (F5H) in Arabidopsis [45]. In the phenylpropanoid pathway, $\mathrm{F} 5 \mathrm{H}$ mainly involved in the biosynthesis of 5-Hydroxy-guaiacyl lignin and syringyl lignin (Fig. 6). Phylogenetic analysis based on P450 gene CYP84A1 of angiosperms showed that E. ferox clustered as the boundary of monocots and eudicots (Fig. 7). The P450 gene sequences of Zea mays, Sorghum bicolor, Ananas comosus, Phoenix dactylifera, Elaeis guineensis, Setaria italica, Oryza sativa, Vitis vinifera, Theobroma cacao, Glycine max, Prunus avium, Cucurbita maxima, Durio zibethinus, Arabidopsis, Nelumbo nucifera etc. in angiosperms together with Ginkgo biloba in gymnosperm as outgroup were used in this study. Consistently with
P450 gene, the NJ tree based on beta-glucosidase gene showed similar result in Additional file 2: Figure S2.

\section{Verification of differential gene expression by quantitative real-time PCR}

To validate the gene expression results derived from the transcriptome data, six DEGs related to nutritional and functional compounds produced during the growth and development of $E$. ferox seed were selected for qRTPCR. Detailed information about the six DEGs is given in Table 3. These DEGs are mainly involved in "starch and sucrose metabolism" (pectinesterase and betaglucosidase), "phenylpropanoid biosynthesis" (cytochrome P450 and phenylalanine ammonia-lyase), and also "carotenoid biosynthesis" (9-cis-epoxycarotenoid dioxygenase and phytoene synthase). As shown in Fig. 8, the six DEGs had very similar expression patterns based on the transcriptome data and qRT-PCR results, which indicates that the results of the transcriptomic analysis are reliable (Additional file 6: Table S4). 


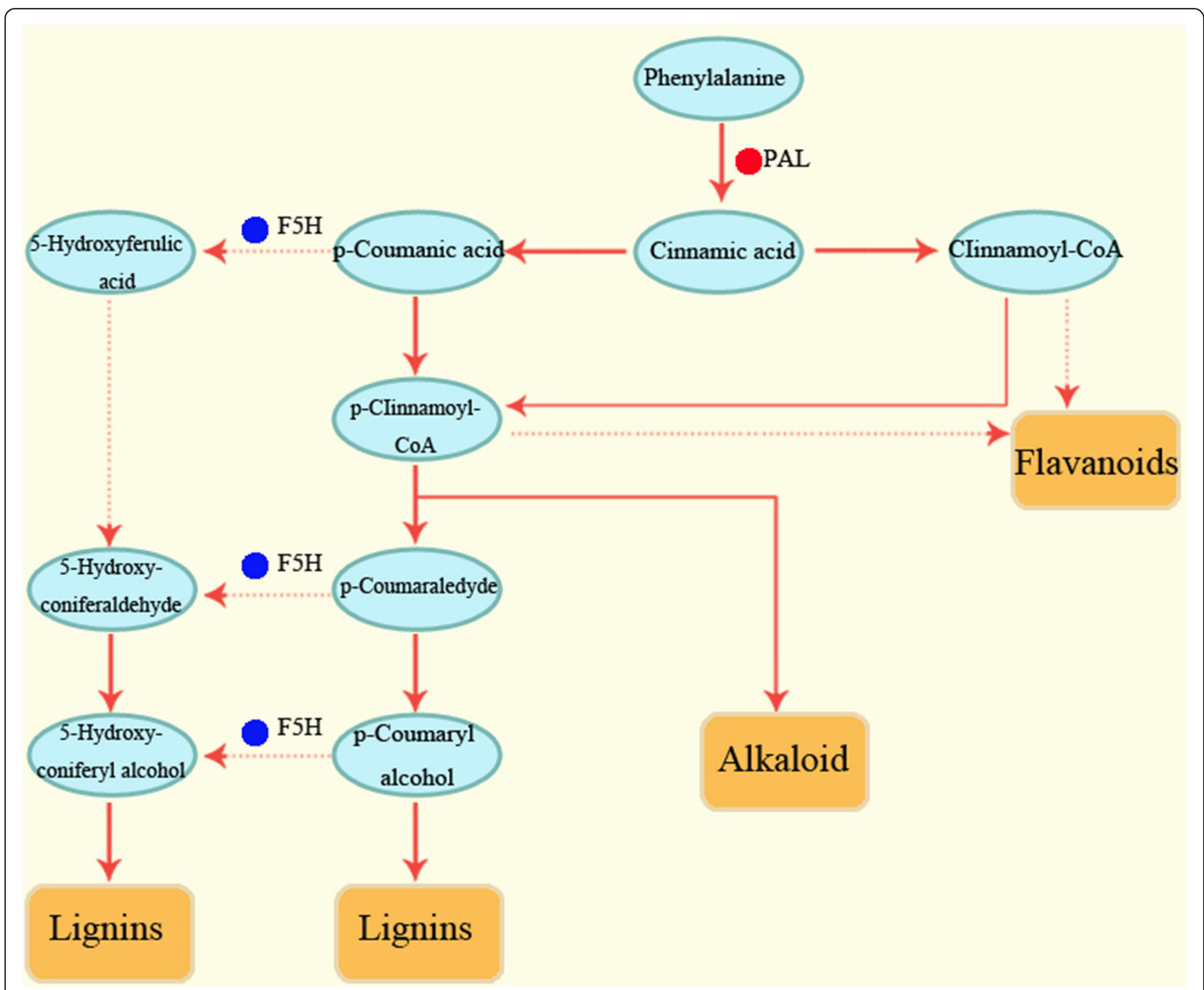

Fig. 6 Simplified scheme of phenylpropanoid biosynthesis. Two key enzymes are PAL (phenylalanine ammonia-lyase) and F5H (ferulate-5-hydroxylase) which called the CYP84A1 gene of cytochrome P450

\section{Discussion}

In recent years, gene expression in an increasing number of horticultural plants has been studied by transcriptomic analysis including cucumber, tomato, water chestnut, lettuce, and peanut [27, 46-49]. With the rapid development of sequencing technology, transcriptomic analysis has become more efficient and cost-effective. Liu et al. [48] reported that 51,792 unigenes with an average length of $849 \mathrm{bp}$ and an N50 length of $1448 \mathrm{bp}$ were obtained from lettuce seed using Trinity assembly software. Yin et al. [49] assembled 59,236 unigenes with a mean length of $751 \mathrm{bp}$ and an N50 length of $1130 \mathrm{bp}$ from transcriptome sequencing of peanut seed. In the present study, the N50 length from our E. ferox seed transcriptome assemblies was greater than in peanut seed but less than in lettuce seed, and all three were generated on the Illumina Hiseq 2000 platform [48, 49]. Undoubtedly, the transcriptome data differs between the three horticultural plant species. In addition, being edible seeds, over 1500 unigenes participate in lipid metabolism in the seven pathways that are related to the accumulation of oil in peanut seed during development [49]. We identified 313 unigenes in the four pathways involved in lipid metabolism in E. ferox seed development. This difference must be due to individual crop attributes; in this case, peanut is an oil crop, and the seeds are rich in oil, but $E$. ferox seed is mainly composed of carbohydrates.

PAL is the key enzyme encoded by a multi-gene family in the biosynthesis of many valuable secondary metabolites, including flavonoid, alkaloid and lignin. There are four members in Arabidopsis [18] and tobacco [19], 


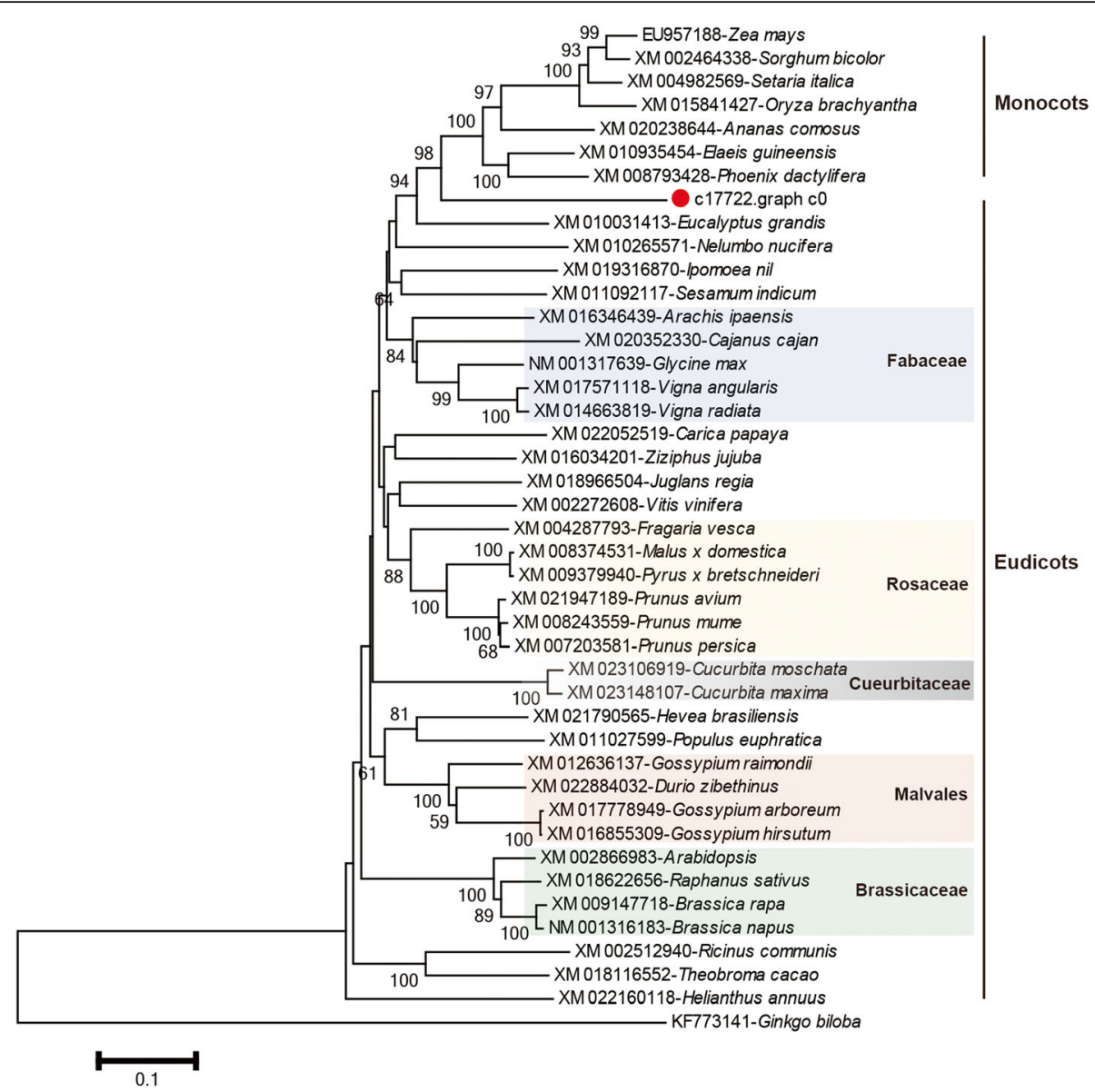

Fig. 7 A phylogenetic tree depicting the relationships among the CYP84A7s in angiosperm. Forty-two CYP84A1 genes from angiosperms with Ginkgo biloba as a outgroup were used in this study

seven members in cucumber [17], and more than twenty copies in tomato [20]. In this study, gene encoding PAL of E. ferox seed is grouped into PAL1. PAL1 has been extensively studied in other plants in order to observe tissuespecific expression and understand secondary metabolites
[14, 17-19]. The expression of PAL1 in E. ferox seed reached its peak at $\mathrm{T} 3$ period, followed by a slight decrease at T4 period. The increasing expression patterns during the maturity of seed influenced the increase of relevant secondary metabolites in phenylpropanoid pathway, which may

Table 3 Oligonucleotide primers used in gene-specific qRT-PCR assays for six DEGs

\begin{tabular}{|c|c|c|c|c|c|c|}
\hline Gene identifier & Gene description & Function & Forward primer (5'-3') & Reverse primer $\left(5^{\prime}-3^{\prime}\right)$ & $\begin{array}{l}\mathrm{Tm} \\
\left({ }^{\circ} \mathrm{C}\right)\end{array}$ & $\begin{array}{l}\text { Product } \\
\text { (bp) }\end{array}$ \\
\hline c44497.graph_c0 & Pectinesterase & $\begin{array}{l}\text { Starch and sucrose } \\
\text { metabolism }\end{array}$ & CGCTGAAGTITGGGAGATG & CCGACCTCCATTAGAAAACG & 54.9 & 113 \\
\hline c50120.graph_c0 & Beta-glucosidase & $\begin{array}{l}\text { Starch and sucrose } \\
\text { metabolism }\end{array}$ & GAACCCTCTTGAGCCTACTTGG & TCCATCCACCGCACTGATAACC & 60.6 & 185 \\
\hline c17722.graph_c0 & Cytochrome P450 & $\begin{array}{l}\text { Phenylpropanoid } \\
\text { biosynthesis }\end{array}$ & TCTCGGTGATGAGTCCTCTGCT & GTTCCGCCATTGCCCATTCTAT & 60.6 & 157 \\
\hline c55946.graph_c2 & $\begin{array}{l}\text { Phenylalanine ammonia- } \\
\text { lyase }\end{array}$ & $\begin{array}{l}\text { Phenylpropanoid } \\
\text { biosynthesis }\end{array}$ & СTTCTCTGGCATTCGGTTCG & ACGATAGCGGCACCAAGTCC & 60.6 & 121 \\
\hline c41810.graph_c1 & $\begin{array}{l}\text { 9-cis-epoxycarotenoid } \\
\text { dioxygenase }\end{array}$ & $\begin{array}{l}\text { Carotenoid } \\
\text { biosynthesis }\end{array}$ & GTCATCCGAAAGCCTTACCTCC & GTGGAAGCAGAAGCAGTCAGGG & 60.6 & 293 \\
\hline c53300.graph_c0 & Phytoene synthase & $\begin{array}{l}\text { Carotenoid } \\
\text { biosynthesis }\end{array}$ & CCTGTTGACATCCAGCCATT & TCATCAGACCGACAGTTCCA & 55.6 & 129 \\
\hline
\end{tabular}




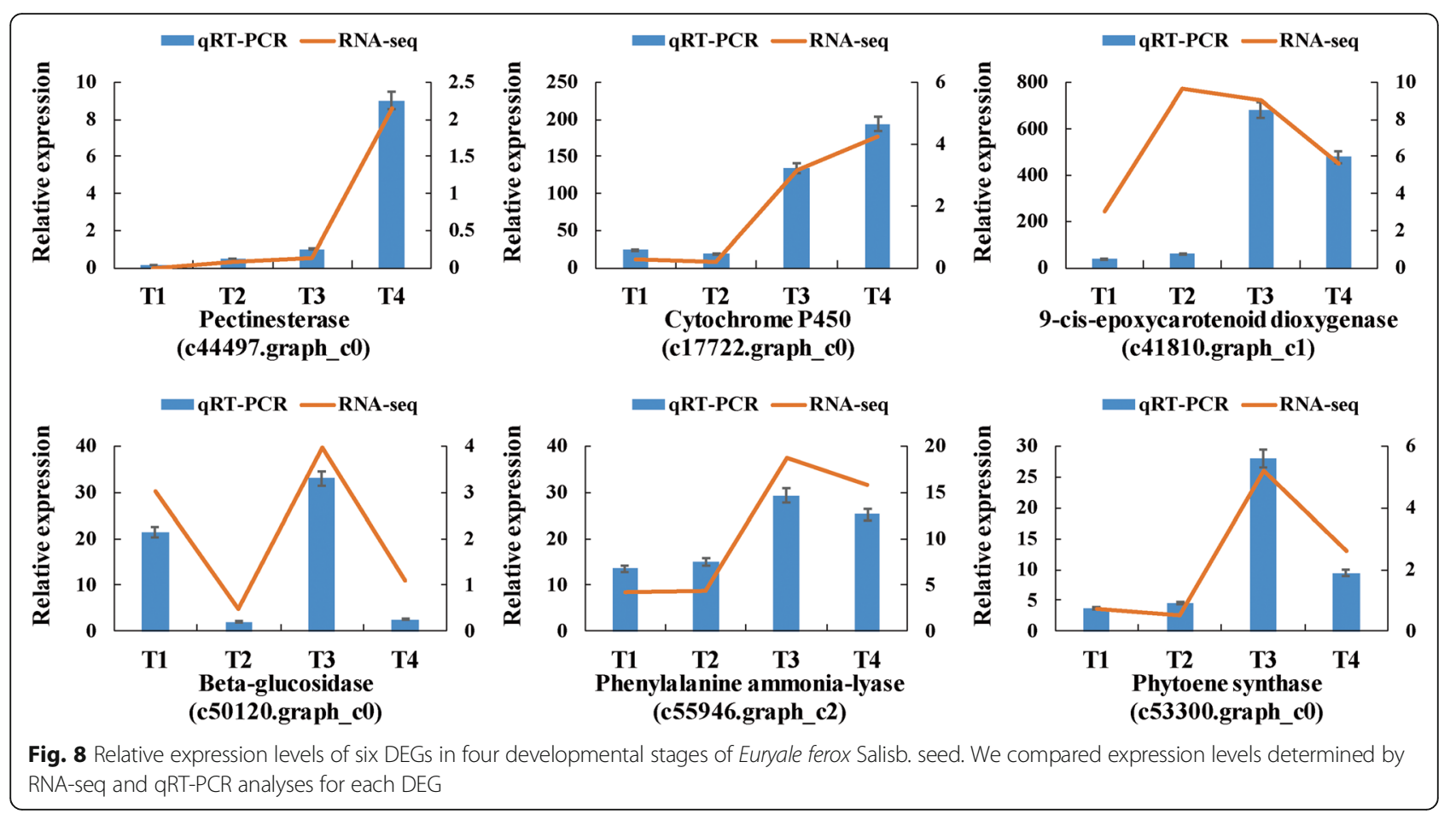

explain the morphological changes and the accumulation of medicinal substances in E. ferox seed. Similar, in the development of the apple (variety 'Jonathan') and strawberry, PAL was related to the anthocyanin accumulation, and anthocyanin is a kind of flavonoid which has lots of health care functions as well as an effect in modification of color $[24,50]$. As shown in Fig. 2, the color of E. ferox seed gradually deepened during the growth and development. This phenotypic change may be regulated by the associated secondary metabolite. Whereas CCPAL1, CCPAL2 and CCPAL3 were verified with the highest expression in the early stage of bean and pericarp in Coffea canephora [51]. The most likely reason was that the synthetic metabolites were different in the growth and development of plants. In fact, the types of metabolite were very rich due to the complexity of the metabolic pathway. Two PALs in Populus tremuloides, PtPAL1 was associated with condensed tannin metabolism and PtPAL2 was involved in monolignol biosynthesis, of which, tannin has remarkable biological and pharmacological activities [52, 53]. In coffee bean, CCPAL1 and CCPAL3 were linked with the accumulation of cholorogenic acids (CGA), whereas CCPAL2 may contribute more importantly to flavonoid accumulation [51]. As a traditional herb, E. ferox seeds are available for human health, so the accumulation of secondary metabolites is closely related to the growth and development of E. ferox seeds. Lignin, as another vital secondary metabolite, is quite crucial for structural integrity of the cell wall and strength and stiffness of the stem [54]. All of the AtPAL1, AtPAL2 and AtPAL4 were associated with lignin biosynthesis in Arabidopsis thaliana [18]. As shown in Fig. 6, there are multiple methods to synthesize lignin during the E. ferox seeds development. In addition to $P A L$, CYP84A1 (F5H) involved in biosynthetic pathway of lignin. The Fig. 2 of four stages in E. ferox seeds shown that, the stiffness of seeds was enhanced. This phenotypic change may be closely related to the accumulation of lignin. During the seed development in wheat, silencing of TaCYP78A3 led to the decrease in wheat seed size and cell proliferation in wheat seed coats, so TaCYP78A3 gene was proved that it can influence the size of seed [26]. The fruit and seeds of E. ferox became obviously larger at the four development stages (Fig. 2). The example in wheat seed can help understand the cellular basis of genes influencing the E. ferox seed development.

For all of the DEGs in E. ferox seed development, the number of DEGs between any two adjacent developmental stages (T1 vs. T2, T2 vs. T3, and T3 vs. T4) was far less than the number found in the T1 vs. T3, T1 vs. T4, and T2 vs. T4 comparisons (Additional file 5: Table S3). This clearly shows constant development in E. ferox seeds. In addition, there were only 21 DEGs in the T3 vs. T4 comparison (Additional file 5: Table S3), which indicated that accumulation of the substances tended to saturate at the later stages of seed development. Above all, the pattern of DEGs 
was coincident with the stages of E. ferox seed growth and development.

\section{Conclusions}

In summary, we generated $313,844,425$ clean reads from the 12 E. ferox seed cDNA libraries using the Illumina Hiseq 2000 platform. 85,006 unigenes with an N50 length of 1399 bp were obtained; of these, 17,769 unigenes were longer than $1 \mathrm{~kb}$. Phenylpropanoid biosynthesis (129 genes annotated with 16 DEGs) involved in the synthesis of important secondary metabolites including flavonoids, lignins and alkaloid. PAL and P450 encoded genes related to this pathway played a vital role in the morphological changes and the accumulation of medicinal components. In the mature stage of E. ferox seed, 21 DEGs indicated that accumulation of the substances tended to saturate. And this pattern is consistent with the growth and development of E. ferox seed.

\section{Additional files}

Additional file 1: Figure S1. KEGG taxonomy of differentially expressed genes. All of the unigenes were assigned to 127 pathways which were divided into five groups: cellular processes, environmental information processing, genetic information processing, metabolism, and organismal systems. (JPG $653 \mathrm{~kb}$ )

Additional file 2: Figure S2. A phylogenetic tree depicting the relationships among the beta-glucosidase genes in angiosperm. Fortynine beta-glucosidase genes from angiosperms with Pinus contorta as a outgroup were used in this study. (JPG $2894 \mathrm{~kb}$ )

Additional file 3: Table S1. The assembled sequences of Euryale ferox were annotated by BLASTX against Arabidopsis thaliana TAIR10. (XLS 490703 kb)

Additional file 4: Table S2. The assembled sequences of Euryale ferox were annotated by BLASTX against rice 7.0 proteomes. (XLS $587887 \mathrm{~kb}$ )

Additional file 5: Tables S3. Differentially expressed genes (DEGs) identified in pairwise comparisons of developmental stages in $E$. ferox seeds. (DOCX $15 \mathrm{~kb}$ )

Additional file 6: Table S4. The FDR of differentially expressed genes (DEGs) identified in pairwise comparisons of developmental stages in $E$. ferox seeds. (DOCX $15 \mathrm{~kb}$ )

\section{Abbreviations \\ CGA: Cholorogenic acids; COG: Clusters of Orthologous Groups: CYP: Cytochrome P450; DEGs: Differentially expressed genes; F5H: Ferulate-5- hydroxylase; FC: Fold Change; FDR: False discovery rate; GO: Gene Ontology; KEGG: Kyoto Encyclopedia of Genes and Genomes; NJ: Neighbor-joining; NR: NCBI non-redundant protein sequences; Ns: Unknown bases; PAL: Phenylalanine ammonia-lyase}

\section{Acknowledgments}

We would like to thank the native English speaking scientists of Elixigen Company (Huntington Beach, California) for editing our manuscript.

\section{Funding}

This work was supported by China Agriculture Research System [grant number CARS-24]. The funding was mainly used for collection of samples, purchase of experimental reagent and modification of the manuscript.

\section{Availability of data and materials}

All Illumina Sequencing data have been deposited in NCBI's Sequence Read Archive (SRA) under accession number SUB2716070 (https://
submit.ncbi.nlm.nih.gov/subs/sra/SUB2716070/overview). The BioProject under accession number SUB2716117 (https://submit.ncbi.n/m.nih.gov/subs/bioproject/ SUB2716117/overview). The BioSample under accession number SUB2718367 (https://submit.ncbi.nlm.nih.gov/subs/biosample/SUB2718367/overview).

\section{Authors' contributions}

$\mathrm{XL}$ analyzed the data, performed the experiments, and drafted the manuscript. $\mathrm{ZH}$ participated in the data analysis and experiments, and helped to draft the manuscript. YLY provided plant materials, participated in experimental design and revised the manuscript. XX and WWW participated in the data analysis and the experiments. $L J L$ conceived the study, participated in its coordination, and helped to draft the manuscript. All of the authors have read and approved the final manuscript.

\section{Ethics approval and consent to participate}

This study has not directly involved humans or animals. The collection of this plant materials comply seriously with the Chinese and international guidelines. Euryale ferox Salisb. has not been listed in the appendices I, II and III of the Convention on the Trade in Endangered Species of Wild Fauna and Flora which was validated from Jan 2th, 2017 (https:// cites.org/eng/app/appendices.php). The initial collector had deposited the voucher specimen in the Chinese Virture Herbarium in July 20th, 1987 with barcode ID: 01604638(http://www.cvh.ac.cn/en/spm/PE/ 01604638). Yunfei Deng undertook the formal identification.

\section{Competing interests}

The authors declare that they have no competing interests.

\section{Publisher's note}

Springer Nature remains neutral with regard to jurisdictional claims in published.

maps and institutional affiliations.

\section{Author details}

${ }^{1}$ School of Horticulture and Plant Protection, Yangzhou University, 48 Wenhui East Road, Yangzhou, Jiangsu Province 225009, People's Republic of China. ${ }^{2}$ Suzhou Vegetable Research Institute, 188 Xitang Road, Suzhou, Jiangsu Province 215008, People's Republic of China.

Received: 12 June 2017 Accepted: 22 April 2018

Published online: 09 May 2018

\section{References}

1. Chen Y, Zhou B. Flora of China. Beijing: Science Press; 1982.

2. Quan ZW, Pan L, Ke WD, Ding Y. Polymorphic microsatellite markers in Euryale ferox Salisb. (Nymphaeaceae). Mol Ecol Resour. 2009;9(1):330-2.

3. Ahmed D, Kumar V, Verma A, Shukla GS, Sharma M. Antidiabetic, antioxidant, antihyperlipidemic effect of extract of Euryale ferox salisb. With enhanced histopathology of pancreas, liver and kidney in streptozotocin induced diabetic rats. SpringerPlus. 2015;4:315.

4. Verma AK, Banerji BK, Chakrabarty D, Datta SK. Studies on Makhana (Euryale ferox Salisbury). Curr Sciindia. 2010;99(6):795-800.

5. Imanishi A, Imanishi J. Seed dormancy and germination traits of an endangered aquatic plant species, Euryale ferox Salisb. (Nymphaeaceae), Aquat Bot. 2014;119:80-3.

6. Ling QZ, Yuan HB, Zhao MX, Wei ZJ, Qi LH, Xu W. Determination of protein and amino acid in seed of Euryale ferox salisb. In wabu lake. Food Res Development. 2009;6:039.

7. Tan SB, Jin T. Nutrtive and health care function and development of Euryale ferox salisb. Food Engineering. 2008;3:8-10.

8. Li SZ. Compendium of Materia Medica. Beijing: People's Medical Publishing House. 2004;33:1902-4

9. Zhao H, Zhao SX, Guillaume D, Sun CQ. New cerebrosides from Euryale ferox. J Nat Prod. 1994;57(1):138-41.

10. Das S, Der P, Raychaudhuri U, Maulik N, Das DK. The effect of Euryale ferox (Makhana), an herb of aquatic origin, on myocardial ischemic reperfusion injury. Mol Cell Biochem. 2006;289(1-2):55-63.

11. Kumar H, Priya P, Singh N, Kumar M, Choudhary BK, Kumar L, et al. RAPD and ISSR marker-based comparative evaluation of genetic diversity among Indian germplasms of Euryale ferox: an aquatic food plant. Appl Biochem Biotech. 2016;180(7):1345-60. 
12. Song CW, Wang SM, Zhou LL, Hou FF, Wang KJ, Han QB, et al. Isolation and identification of compounds responsible for antioxidant capacity of Euryale ferox seeds. J Agr Food Chem. 2011;59(4):1199-204.

13. Wu CY, Chen R, Wang XS, Shen B, Yue W, Wu QA. Antioxidant and antifatigue activities of phenolic extract from the seed coat of Euryale ferox salisb. And identification of three phenolic compounds by LC-ESI-MS/MS. Molecules. 2013;18(9):11003-21.

14. Hou X, Shao F, Ma Y, Lu S. The phenylalanine ammonia-lyase gene family in Salvia miltiorrhiza: genome-wide characterization, molecular cloning and expression analysis. Mol Biol Rep. 2013;40(7):4301-10.

15. Du H, Ran F, Dong HL, Wen J, Li JN, Liang Z. Genome-wide analysis, classification, evolution, and expression analysis of the cytochrome P450 93 family in land plants. PLoS One. 2016;11(10):e0165020.

16. Nielsen KA, Møller BL. Cytochrome P450s in Plants. In: Ortiz de Montellano P.R. (eds) Cytochrome P450. Boston: Springer; 2005.

17. Shang QM, Li L, Dong CJ. Multiple tandem duplication of the phenylalanine ammonia-lyase genes in Cucumis sativus L. Planta. 2012;236(4):1093-105.

18. Raes J, Rohde A, Christensen JH, Peer YVD, Boerjan W. Genome-wide characterization of the lignification toolbox in Arabidopsis. Plant Physiol. 2003;133(3):1051-71.

19. Reichert Al, He XZ, Dixon RA. Phenylalanine ammonia-lyase (PAL) from tobacco (Nicotiana tabacum): characterization of the four tobacco PAL genes and active heterotetrameric enzymes. Biochem J. 2009;424(2):233-42.

20. Chang A, Lim MH, Lee SW, Robb EJ, Nazar RN. Tomato phenylalanine ammonia-lyase gene family, highly redundant but strongly underutilized. J Biol Chem. 2008;283(48):33591-601.

21. Nelson DR, Schuler MA, Paquette SM, Werck-Reichhart D, Bak S. Comparative genomics of rice and Arabidopsis. Analysis of 727 cytochrome P450 genes and pseudogenes from a monocot and a dicot. Plant Physiol. 2004:135(2):756-72.

22. Nelson DR. The cytochrome P450 homepage. Hum Genomics. 2009;4(1):59-65.

23. Wanner LA, Li G, Ware D, Somssich IE, Davis KR. The phenylalanine ammonia-lyase gene family in Arabidopsis thaliana. Plant Mol Biol. 1995; 27(2):327-38.

24. Pombo MA, Martinez GA, Civello PM. Cloning of FaPAL6 gene from strawberry fruit and characterization of its expression and enzymatic activity in two cultivars with different anthocyanin accumulation. Plant Sci. 2011; 181(2):111-8.

25. Ivamoto ST, Sakuray LM, Ferreira LP, Kitzberger CSG, Scholz MBS, Pot D, et al. Diterpenes biochemical profile and transcriptional analysis of cytochrome p450s genes in leaves, roots, flowers, and during Coffea arabica L. fruit development. Plant Physiol Bioch. 2017;111:340-7.

26. Ma M, Wang Q, Li Z, Cheng H, Li Z, Liu X, et al. Expression of TaCYP78A3, a gene encoding cytochrome P450 CYP78A3 protein in wheat (Triticum aestivum L.), affects seed size. Plant J Cell Mol Biol. 2015;83(2):312-25.

27. Cheng LB, Li SY, Chen SN, Wang Y, Yu MZ, Chen XH, et al. Transcriptome analysis of gene expression during Chinese water chestnut storage organ formation. PLoS One. 2016;11(10):e0164223.

28. Yu RG, Xu L, Zhang W, Wang Y, Luo XB, Wang RH, et al. De novo taproot transcriptome sequencing and analysis of major genes involved in sucrose metabolism in radish (Raphanus sativus L.). Front Plant Sci. 2016;7:585.

29. Grabherr MG, Haas BJ, Yassour M, Levin JZ, Thompson DA, Amit I, et al. Fulllength transcriptome assembly from RNA-Seq data without a reference genome. Nat Biotechnol. 2011;29(7):644-52.

30. Deng YY, Li JQ, Wu SF, Zhu YP, Chen YW, He FC. Integrated nr database in protein annotation system and its localization. Comput Eng. 2006:32:71-4.

31. Tatusov RL, Galperin MY, Natale DA, Koonin EV. The COG database: a tool for genome-scale analysis of protein functions and evolution. Nucleic Acids Res. 2000;28(1):33-6

32. Apweiler R, Bairoch A, Wu CH, Barker WC, Boeckmann B, Ferro S, et al. UniProt: the universal protein knowledgebase. Nucleic Acids Res. 2004;32:D115-9.

33. Kanehisa M, Goto S, Kawashima S, Okuno Y, Hattori M. The KEGG resource for deciphering the genome. Nucleic Acids Res. 2004:32:D277-80.

34. Ashburner M, Ball CA, Blake JA, Botstein D, Butler H, Michael Cherry J, et al. Gene ontology: tool for the unification of biology. Nature Genet. 2000;25:25-9.

35. Iseli C, Jongeneel CV, Bucher P. ESTScan: a program for detecting, evaluating, and reconstructing potential coding regions in EST sequences. Proceedings. International Conference on Intelligent Systems for Molecular Biology. 1999;99:138-58.
36. Langmead B, Trapnell C, Pop M, Salzberg SL. Ultrafast and memory-efficient alignment of short DNA sequences to the human genome. Genome Biol. 2009;10:R25

37. Li B, Dewey CN. RSEM: accurate transcript quantification from RNA-Seq data with or without a reference genome. BMC Bioinformatics. 2011;12:323.

38. Trapnell C, Williams BA, Pertea G, Mortazavi A, Kwan G, van Baren MJ, et al. Transcript assembly and quantification by RNA-Seq reveals unannotated transcripts and isoform switching during cell differentiation. Nat Biotechnol. 2010;28(5):511-5.

39. Leng N, Dawson JA, Thomson JA, Ruotti V, Rissman Al, Smits BMG, et al EBSeq: an empirical Bayes hierarchical model for inference in RNA-seq experiments. Bioinformatics. 2013;29(8):1035-43.

40. Kumar S, Stecher G, Tamura K. Mega7: molecular evolutionary genetics analysis version 7.0 for bigger datasets. Mol Biol Evol. 2016;33(7):1870-4.

41. Liang DN, Liu M, Hu QJ, He M, Qi XH, Xu Q, et al. Identification of differentially expressed genes related to aphid resistance in cucumber (Cucumis sativus L.). Sci Rep. 2015;5:9645.

42. Wu T, Qin ZW, Zhou XY, Feng Z, Du YL. Transcriptome profile analysis of floral sex determination in cucumber. J Plant Physiol. 2010;167(11):905-13.

43. Mach J. Unpureeing the tomato: layers of information revealed by microdissection and high-throughput transcriptome sequencing. Plant Cell. 2011;23(11):3668.

44. Zhang CS, Zhang HW, Zhan ZX, Liu BJ, Chen ZT, Liang Y. Transcriptome analysis of sucrose metabolism during bulb swelling and development in onion (Allium cepa L.). Front. Plant Sci. 2016;7:1425.

45. Ruegger M, Meyer K, Cusumano JC, Chapple C. Regulation of ferulate-5hydroxylase expression in Arabidopsis in the context of sinapate ester biosynthesis. Plant Physiol. 1999;119(1):101-10.

46. Ando K, Grumet R. Transcriptional profiling of rapidly growing cucumber fruit by 454-pyrosequencing analysis. J Am Soc Hortic Sci. 2010;135(4):291-302.

47. Tang XF, Tang ZZ, Huang SX, Liu JK, Liu J, Shi W, et al. Whole transcriptome sequencing reveals genes involved in plastid/chloroplast division and development are regulated by the HP1/DDB1 at an early stage of tomato fruit development. Planta. 2013;238(5):923-36.

48. Liu SJ, Song SH, Wang WQ, Song SQ. De novo assembly and characterization of germinating lettuce seed transcriptome using Illumina paired-end sequencing. Plant physiol bioch. 2015;96:154-62.

49. Yin DM, Wang Y, Zhang XG, Li HM, Lu X, Zhang JS, et al. De novo assembly of the peanut (Arachis hypogaea L.) seed transcriptome revealed candidate unigenes for oil accumulation pathways. PLoS One. 2013;8(9):UNSP e73767.

50. Wang HQ, Arakawa O, Motomura Y. Influence of maturity and bagging on the relationship between anthocyanin accumulation and phenylalanine ammonialyase (PAL) activity in 'Jonathan' apples. Postharvest Biol Tec. 2000;19(2):123-8.

51. Maud L, Venkataramaiah M, James MC, Michel $R$, Dominique $C$, Nathalie $C$ et al. Characterization, high-resolution mapping and differential expression of three homologous PAL genes in Coffea canephora Pierre (Rubiaceae). Planta. 2012;236(1):313-26.

52. Kao YY, Harding SA, Tsai CJ. Differential expression of two distinct phenylalanine ammonia-lyase genes in condensed tannin-accumulating and lignifying cells of quaking aspen. Plant Physiol. 2002;130(2):796-807.

53. Okuda T. Systematics and health effects of chemically distinct tannins in medicinal plants. Phytochemistry. 2005;66(17):2012-31.

54. Boerjan W, Ralph J, Baucher M. Lignin biosynthesis. Annu Rev Plant Biol. 2003:54(1):519-46

Ready to submit your research? Choose BMC and benefit from

- fast, convenient online submission

- thorough peer review by experienced researchers in your field

- rapid publication on acceptance

- support for research data, including large and complex data types

- gold Open Access which fosters wider collaboration and increased citations

- maximum visibility for your research: over $100 \mathrm{M}$ website views per year

At BMC, research is always in progress.

Learn more biomedcentral.com/submissions 